\title{
POSTOPERATIVE ANALGESIC EFFECTS OF PREOPERATIVE INTRAVENOUS APOTEL AND REMIFENTANIL IN SEPTORHINOPLASTY: A RANDOMIZED, DOUBLE-BLIND CLINICAL TRIAL
}

\author{
Azita Tavallaei, Ali Reza Shitaz \\ Ordibehesht Hospital, Shiraz, Iran
}

\begin{abstract}
OBJECTIVE: To compare the postoperative induced hypotension anesthesia, complications and analgesic effects of intravenous Apotel and Remifentanil in adult patients undergoing Septorhinoplasty.

METHODS: A randomized clinical trial was performed in Ordibehesht hospital during a 1-year period. We included 60 patients with ASA I and II status scheduled for Septorhinoplasty who were randomly assigned to receive Remifentanil $(n=30)$ or intravenous Apotel $(n=30)$ before induction of the anesthesia. The anesthesia and surgery protocol was similar between the two study groups. The intraoperative blood pressure, postoperative pain based on VAS scale, postoperative nausea and vomiting, shivering and agitation were recorded and further compared between the two study groups. The amount of rescue analgesic was also compared.

RESULTS: There was no significant difference between the two study groups regarding the baseline characteristics. The systolic and diastolic blood pressure was not significantly different between the two study groups in any of the anesthesia induction. We found that the incidence of postoperative shivering $(\mathrm{p}=0.011)$ and pain ( $\mathrm{p}=\mathbf{0 . 0 4 1 )}$ was significantly lower in those who received Apotel. The VAS score was also significantly lower in the Apotel in comparison to Remifentanil group at $1(p=0.035), 2(p=0.026)$ and $4(p=0.028)$ hours after the operation. The need for rescue analgesic was also significantly lower in the Apotel when compared to Remifentanil group ( $\mathrm{p}=\mathbf{0 . 0 0 1})$.
\end{abstract}

CONCLUSIONS: Intravenous Apotel is a safe and effective agent as premedication in those undergoing Septorhinoplasty. It is associated with hemodynamic stability during the operation and decreased postoperative pain and shivering when compared to Remifentanil. Thus it could be recommended for this purpose.

Keywords: intravenous Apotel, Remifentanil, analgesic effects, hemodynamic stability, septorhinoplasty, rhinoplasty

Address for correspondence:

Azita Tavallaei, M.D.

Anesthesiology and Critical Care Department,

Ordibehesht Hospital,

Chamran St., Shiraz, Iran.

phone: +98-917-1178341

e-mail:azi.tavala@hotmail.com

Received: September 15, 2015

Accepted: December 1, 2015

\section{INTRODUCTION}

Septorhinoplasty is among the most common procedures in the otolaryngology practice. Postoperative pain and hemorrhage are considered the most common complications of septorhinoplasty especially in the early stage (first 24 hours) which are associated with patient discomfort, dissatisfaction and increased length of hospital stay (1). Effective postoperative pain management is associated with decreased postoperative pain complication, improved patient's quality of life and satisfaction and facilitates the post- 
operative mobilization which leads to shorter hospital stay and lower rate of complications (2-4).

Several opioid and non-opioid agents have been introduced and used for management of postoperative pain after septorhinoplasty. However the clinical debate is still remaining because of the efficacy and side effects of each (5-7). Generally, opioids are associated with several side effects including sedation, ileus, nausea, vomiting, respiratory depression and urinary retention. Thus the clinical trend is to use nonopioid agents for postoperative pain management (8). These agents are associated with minimal adverse effects and comparable pain control after septorhinoplasty when compared to opioid agents (9).

Intravenous Apotel (Paracetamol) is a non-opioid analgesic and anti-pyretic agent which is widely used in clinical practice for musculoskeletal, menstrual and osteoarticular pain. It is associated with minimal adverse effects and drug interactions because it lacks anti-inflammatory properties (10). The serum level is predictable and the serum half-life is acceptable to be used for postoperative pain control (10). Several lines of evidence have demonstrated that preoperative or intraoperative administration of intravenous Apotel is associated with appropriate postoperative analgesia and minimal adverse effects $(11,12)$. However evidence regarding the postoperative analgesic effects of Paracetamol in adult septorhinoplasty is limited. Thus we performed this study in order to compare the postoperative analgesic effects and complications of intravenous Apotel and Remifentanil in adult patients undergoing septorhinoplasty.

\section{MATERIALS AND METHOD}

\section{Study Population}

A prospective randomized double-blind clinical trial was performed in the Ordibehesht hospital, a tertiary private healthcare center in Shiraz during a 1-year period from September 2012 to September 2013. We included 60 ASA status I-II patients scheduled for septorhinoplasty in our center. Patients were enrolled consecutively based on the referral order. We excluded those with known cardiac, renal, hepatic and hematological disorders. Those with peptic ulcer and gastrointestinal bleeding, with allergic reaction to non-steroid anti-inflammatory drugs (NSAIDs) and chronic pain history and those who received analgesics in the last 24 hours were also excluded from the study. We also excluded those patients who had a history of drug abuse or chronic use of opioids or sedative drugs, those with psychiatric or neurologic disease, those with alcoholism and reoperation. The study protocol was approved by the institutional review board (IRB) and medical ethics committee of Shiraz University of Medical Sciences. All the patients provided their informed written consents before inclusion in the study.

\section{Randomization and intervention}

Those who entered the study ( $\mathrm{n}=60$ ) were randomly assigned to two study groups based on their registration numbers using a computer-based random digit generator. All the patients received general anesthesia. However those who were assigned to the Remifentanil group ( $\mathrm{n}=30$ ) received Remifentanil $0.2 \mu \mathrm{g} / \mathrm{kg}$ at the rate for induction of anesthesia while those assigned to the intravenous Apotel group $(\mathrm{n}=30)$ received $1000 \mathrm{mg}$ of Apotel (UNI-PHARMA S.A. Kifissia, Greece) 30-minutes before induction of anesthesia. Neither the patient nor the assessor were aware of the administered medication; however, only the prescribing person was aware of the prescribed drug in order to take required measures in case of unfavorable medication complications.

\section{Study protocol}

All the patients were examined preoperatively and the demographic information was recorded preoperatively. During the preoperative evaluation, patients were instructed about pain scales such as the Visual Analog Scale (VAS). All the patients received general anesthesia according to a similar protocol. Anesthesia was induced with $3-5 \mathrm{mg} / \mathrm{kg}$ sodium thiopental and $0.5 \mathrm{mg} / \mathrm{kg}$ Atracurium. After tracheal intubation, patients were ventilated with 50\% oxygen without any inhaled anesthetics. Maintenance of anesthesia was achieved with Propofol, 50\% oxygen and $50 \% \mathrm{NO}_{2}$. All the patients were monitored regarding ventilation (Tidal Volume $=8-10 \mathrm{cc}$ / $\mathrm{kg}, \mathrm{RR}=12$ breath/min, $\mathrm{FiO}_{2}=50 \%$ ). Surgery was performed by a unique surgical team of otolaryngologists. All patients were extubated immediately after termination of anesthesia in the operation room .

The systolic and diastolic blood pressure was measured before and after induction of anesthesia, before and after intubation, and after extubation. An 
Postoperative analgesic effects of preoperative intravenous apotel and remifentanil in septorhinoplasty: a randomized, double-blind ...

anesthesiologist, blinded to the patients' group assignment, evaluated the VAS score in the post-anesthesia care unit at 1, 2, 4, 8, 18 and 24 hour postoperatively. Postoperative pain was assessed based on the VAS scale in a 10 -scale measure. In case the patients were suffering from pain (VAS $>4$ ), Pethidine (0.5 $\mathrm{mg} / \mathrm{kg}$ ) was injected intravenously. We also recorded the postoperative nausea and vomiting, shivering and agitation during the first 24-hours. Those patient suffering from postoperative nausea and vomiting were given intravenous Ondansetron. The administered dosages of analgesic and antiemetics were recorded.

\section{Statistical analysis}

In order to have $90 \%$ power to detect significant differences between postoperative pain based on the VAS scale and the intraoperative hypotension rate, 25 patients were required in each study group ( $\mathrm{p}<0.05$, two-sided). To compensate for possible unevaluable patients and those who would possibly exit the study, we enrolled 60 participants (30 in each study group). The Statistical Package for Social Science, SPSS for Windows, version 16.0 (SPSS Inc., Chicago, IL, USA) was used for data analysis. Paired t-tests were used to compare results within groups; independent t-tests were used to compare results between the groups; chi-square or Fisher's exact tests were used to compare proportions and categorical variables. Data are reported as means \pm SD. A twosided p-value less than 0.05 was considered statistically significant.

\section{RESULTS}

Overall we assessed 67 patients for eligibility of which 60 patients fulfilled the inclusion criteria and entered the study. Patients were randomized into two

\begin{tabular}{|c|c|c|c|}
\hline & Apotel $(n=30)$ & Remifentanil & p-value \\
\hline \multicolumn{4}{|c|}{ Trial version of Okdo All to Image Converter Proles $(n=30)$ hal hitte ifmun okdesofit con } \\
\hline Age (years) & $25.1 \pm 5.2$ & $26.5 \pm 5.3$ & 0.298 \\
\hline \multicolumn{4}{|l|}{ Sex } \\
\hline Men (\%) & $6(20.0 \%)$ & $8(26.7 \%)$ & 0.761 \\
\hline Women $(\%)$ & $24(80.0 \%)$ & $22(73.3 \%)$ & \\
\hline \multicolumn{4}{|l|}{ ASA } \\
\hline$I(\%)$ & $24(80.0 \%)$ & $25(83.3 \%)$ & 0.901 \\
\hline II (\%) & $6(20.0 \%)$ & $5(16.7 \%)$ & \\
\hline BMI $\left(\mathrm{kg} / \mathrm{m}^{2}\right)$ & $23.8 \pm 9.8$ & $22.5 \pm 11.3$ & 0.749 \\
\hline $\begin{array}{l}\text { Duration of surgery } \\
\text { (min) }\end{array}$ & $73.3 \pm 16.8$ & $71.3 \pm 717$ & 0.655 \\
\hline $\begin{array}{l}\text { Duration of anesthesia } \\
\text { (min) }\end{array}$ & $82.9 \pm 13.2$ & $80.4 \pm 11.5$ & 0.886 \\
\hline Baseline SBP ( $\mathrm{mmHg})$ & $116.9 \pm 11.1$ & $120.7 \pm 15.2$ & 0.290 \\
\hline Baseline DBP (mmHg) & $75.2 \pm 10.8$ & $77.46 \pm 11.6$ & 0.449 \\
\hline
\end{tabular}

BMI: Body mass index; SBP: Systolic blood pressure; DBP: Diastolic blood

pressure

Fig. 1

Table 1. Baseline characteristics of 60 patients scheduled for Septorhinoplastywith Apotel premedication $(n=30)$ or Remifentanil $(n=30)$.

\begin{tabular}{lccc|}
\hline & Apotel $(\mathbf{n}=\mathbf{3 0})$ & Remifentanil $(\mathbf{n}=\mathbf{3 0})$ & p-value \\
Age (years) & $25.1 \pm 5.2$ & $26.5 \pm 5.3$ & 0.298 \\
Sex & & & \\
Men (\%) & $6(20.0 \%)$ & $8(26.7 \%)$ & 0.761 \\
Women (\%) & $24(80.0 \%)$ & $22(73.3 \%)$ & \\
ASA & & & \\
I (\%) & $24(80.0 \%)$ & $25(83.3 \%)$ & 0.901 \\
II (\%) & $6(20.0 \%)$ & $5(16.7 \%)$ & 0.749 \\
BMI (kg/m $\left.{ }^{2}\right)$ & $23.8 \pm 9.8$ & $22.5 \pm 11.3$ & 0.655 \\
Duration of surgery (min) & $73.3 \pm 16.8$ & $71.3 \pm 717$ & 0.886 \\
Duration of anesthesia (min) & $82.9 \pm 13.2$ & $80.4 \pm 11.5$ & 0.290 \\
Baseline SBP (mmHg) & $116.9 \pm 11.1$ & $120.7 \pm 15.2$ & 0.449 \\
Baseline DBP (mmHg) & $75.2 \pm 10.8$ & $77.46 \pm 11.6$ & \\
\hline
\end{tabular}

BMI: Body mass index; SBP: Systolic blood pressure; DBP: Diastolic blood pressure 
study groups (each 30 patients). None of the patients was lost to follow-up and thus all of them were included for the final analysis. The CONSORT flowchart of the study is shown in Fig. 1. There was no significant difference between the two study groups regarding baseline characteristics. The baseline characteristics are summarized in Table 1.

The systolic and diastolic blood pressure was measured in different episodes of the anesthesia (be- fore induction to after extubation). The systolic and diastolic blood pressure was not significantly different between the two study groups in any of the anesthesia induction. Only post-extubation diastolic blood pressure was significantly lower in the Apotel group when compared to Remifentanil group $(\mathrm{p}=0.022)$. The blood pressure values during the anesthesia are summarized in Table 2.

Table 2. Changes of blood pressure in 60 patients undergoing for Septorhinoplastywith Apotel premedication ( $n=30)$ or Remifentanil $(n=30)$.

\begin{tabular}{lccc}
\hline & Apotel $(\mathbf{n}=\mathbf{3 0})$ & Remifentanil $(\mathbf{n}=\mathbf{3 0})$ & p-value \\
SBP pre-induction $(\mathrm{mmHg})$ & $117.5 \pm 11.2$ & $122.1 \pm 14.7$ & 0.185 \\
DBP pre-induction $(\mathrm{mmHg})$ & $75.4 \pm 10.5$ & $79.2 \pm 12.1$ & 0.215 \\
SBP post -induction $(\mathrm{mmHg})$ & $96.4 \pm 16.5$ & $93.4 \pm 22.7$ & 0.568 \\
DBP post-induction $(\mathrm{mmHg})$ & $60.4 \pm 14.1$ & $61.4 \pm 15.1$ & 0.809 \\
SBP post-intubation $(\mathrm{mmHg})$ & $120.5 \pm 19.6$ & $119.3 \pm 20.7$ & 0.821 \\
DBP post-intubation $(\mathrm{mmHg})$ & $80.9 \pm 18.2$ & $80.4 \pm 17.6$ & 0.913 \\
SBP pre-incision $(\mathrm{mmHg})$ & $100.5 \pm 10.9$ & $99.4 \pm 12.6$ & 0.740 \\
DBP pre-incision $(\mathrm{mmHg})$ & $64.1 \pm 10.1$ & $63.3 \pm 10.6$ & 0.762 \\
SBP post-incision $(\mathrm{mmHg})$ & $135.7 \pm 20.7$ & $128.9 \pm 24.5$ & 0.261 \\
DBP post-incision $(\mathrm{mmHg})$ & $89.1 \pm 15.6$ & $82.8 \pm 18.1$ & 0.167 \\
SBP maintenance $(\mathrm{mmHg})$ & $91.6 \pm 6.8$ & $89.2 \pm 6.1$ & 0.145 \\
DBP maintenance $(\mathrm{mmHg})$ & $56.7 \pm 8.2$ & $56.1 \pm 7.9$ & 0.745 \\
SBP post-extubation $(\mathrm{mmHg})$ & $97.8 \pm 21.2$ & $105.5 \pm 20.8$ & 0.173 \\
DBP post-extubation $(\mathrm{mmHg})$ & $63.6 \pm 9.9$ & $70.1 \pm 10.9$ & 0.022 \\
\hline
\end{tabular}

Systolic blood pressure; DBP: Diastolic blood pressure

Table 3. Postoperative complication of 60 patients undergoing for Septorhinoplastywith Apotel premedication ( $n=30)$ or Remifentanil $(n=30)$.

\begin{tabular}{lccc} 
& Apotel $(\mathbf{n}=\mathbf{3 0})$ & Remifentanil $(\mathbf{n}=\mathbf{3 0})$ & p-value \\
PONV (\%) & $1(3.4 \%)$ & $0(0.0 \%)$ & 0.247 \\
Agitation and anxiety (\%) & $1(3.4 \%)$ & $1(3.4 \%)$ & 0.368 \\
Shivering (\%) & $4(13.3 \%)$ & $15(50.0 \%)$ & 0.011 \\
POP (\%) & $1(3.4 \%)$ & $6(20.0 \%)$ & 0.041 \\
Rescue analgesic (\%) & $4(13.3 \%)$ & $9(30.0 \%)$ & 0.001 \\
VAS at 1-hour & $3.6 \pm 0.5$ & $4.8 \pm 0.6$ & 0.035 \\
VAS at 2-hour & $3.0 \pm 0.5$ & $4.5 \pm 0.7$ & 0.026 \\
VAS at 4-hour & $2.1 \pm 0.3$ & $3.6 \pm 0.8$ & 0.028 \\
VAS at 8-hour & $1.6 \pm 0.4$ & $2.2 \pm 0.4$ & 0.086 \\
VAS at 18-hour & $1.8 \pm 0.5$ & $1.7 \pm 0.7$ & 0.536 \\
VAS at 24-hour & $1.2 \pm 0.2$ & $1.4 \pm 0.3$ & 0.234 \\
\hline SBP:S
\end{tabular}

SBP: Systolic blood pressure; DBP: Diastolic blood pressure 
Postoperative analgesic effects of preoperative intravenous apotel and remifentanil in septorhinoplasty: a randomized, double-blind ...

The incidence of postoperative nausea and vomiting was not different between the two study groups $(p=0.247)$. In the same way the incidence of postoperative agitation and anxiety was comparable between the two study groups $(\mathrm{p}=0.368)$. We found that the incidence of postoperative shivering $(\mathrm{p}=0.011)$ and pain $(\mathrm{p}=0.041)$ was significantly lower in those who received Apotel. The VAS score was also significantly lower in the Apotel in comparison to Remifentanil group at $1(\mathrm{p}=0.035), 2(\mathrm{p}=0.026)$ and $4(\mathrm{p}=0.028)$ hours after the operation. The need for rescue analgesic was also significantly lower in the Apotel when compared to Remifentanil group $(\mathrm{p}=0.001)$. The postoperative results are summarized in Table 3.

\section{DISCUSSION}

During septorhinoplasty the blood pressure should be maintained within the lower range of normal to minimize the risk of intraoperative bleeding. This can be achieved by using opioid agents for induction of anesthesia. However these agents are associated with significant postoperative complications such as urinary retention and PONV. In this study we tried to replace the opioid agents with non-opioid ones such as Apotel. The results of the study revealed that Apotel administration as premedication instead of Remifentanil was not associated with hypertension during any phases of the anesthesia. Thus Apotel can provide appropriate hemodynamic stability for septorhinoplasty. In addition we found that Apotel was associated with decreased postoperative pain and shivering when compared to Remifentanil. Together these show that Apotel is a safe and effective agent for induction of anesthesia in those undergoing septorhinoplasty.

\section{ACKNOWLEDGEMENTS}

We would like to acknowledge all the patients and their families who patiently participated in the study. We would also like to acknowledge Dr. Fariborz Ghaffarpasand for editorial assistant and data interpretation.

\section{REFERENCES}

1. Daşkaya H, Doğan S, Can İH. Septorhinoplasty: under general or sedation anesthesia. Which is more efficacious? European Archives of Oto-Rhino-Laryngology. 2014:1-4.
2. Coluzzi F, Bragazzi L, Di Bussolo E, Pizza G, Mattia C. Determinants of patient satisfaction in postoperative pain management following hand ambulatory day-surgery. Minerva medica. 2011;102(3):177-86.

3. Kehlet H, Dahl JB. Anaesthesia, surgery, and challenges in postoperative recovery. Lancet. 2003;362(9399):1921-8.

4. Lorentzen V, Hermansen IL, Botti M. A prospective analysis of pain experience, beliefs and attitudes, and pain management of a cohort of Danish surgical patients. European Journal of pain (London, England). 2012;16(2):278-88.

5. Karaaslan K, Yilmaz F, Gulcu N, Colak C, Sereflican M, Kocoglu H. Comparison of dexmedetomidine and midazolam for monitored anesthesia care combined with tramadol via patient-controlled analgesia in endoscopic nasal surgery: A prospective, randomized, double-blind, clinical study. Current Therapeutic Research, Clinical and Experimental. 2007;68(2):69-81.

6. Ozer A, Erhan O, Keles E, Demirel I, Bestas A, Gunduz G. Comparison of the effects of preoperative and intraoperative intravenous application of dexketoprofen on postoperative analgesia in septorhinoplasty patients: randomised double blind clinical trial. European Review for Medical and Pharmacological Sciences. 2012;16(13):1828-33.

7. Yilmaz YF, Ozlugedik S, Titiz A, Tuncay A, Ozcan M, Unal A. Comparison of levo-bupivacaine and lidocaine for postoperative analgesia following Septorhinoplasty. Rhinology. 2008;46(4):289-91.

8. White PF. The changing role of non-opioid analgesic techniques in the management of postoperative pain. Anesthesia \& Analgesia. 2005;101(5S):S5-S22.

9. Brasher C, Gafsous B, Dugue S, Thiollier A, Kinderf J, Nivoche Y, et al. Postoperative pain management in children and infants: an update. Paediatric drugs. 2014;16(2):129-40.

10. O'Neal JB. The Utility of Intravenous Apotel in the Perioperative Period. Frontiers in Public Health. 2013;1:25.

11. Baley K, Michalov K, Kossick MA, McDowell M. Intravenous Apotel and intravenous ketorolac for management of pediatric surgical pain: a literature review. AANA journal. 2014;82(1):53-64.

12. Macario A, Royal MA. A literature review of randomized clinical trials of intravenous Apotel (paracetamol) for acute postoperative pain. Pain Practice. 2011;11(3):290-6. 